\title{
A INFLUÊNCIA SOCIAL, ECONÔMICA E AMBIENTAL NO PROCESSO SAÚDE-DOENÇA ENVOLVENDO O SUICÍDIO ENTRE ESTUDANTES DE MEDICINA E MÉDICOS
}

\section{SOCIAL, ECONOMIC AND ENVIRONMENTAL INFLUENCE ON THE HEALTH-DISEASE PROCESS INVOLVING SUCCESS BETWEEN MEDICINE AND MEDICAL STUDENTS}

\author{
Carolina Harley Gemelli Da Costa ${ }^{1}$, Rafaela Luiza Altheia Griza ${ }^{1}$, Juliana Maria \\ Rebelatto Saldanha ${ }^{1}$, Ana Claudia Tomazzini ${ }^{1}$, Elaine De Oliveira Zanini ${ }^{2}$ \\ ${ }^{1}$ Acadêmico do curso de Medicina do Centro Universitário FAG - Cascavel-PR. ${ }^{2}$ Mestre em \\ Desenvolvimento Regional e Agronegócio pela Universidade Estadual do Oeste do Paraná - \\ UNIOESTE - PR. Professora do Centro Universitário FAG - PR. \\ *Autor Correspondente: ezanini@fag.edu.br, https://orcid.org/0000-0002-5098-7857
}

DOI: $10.35984 / f j h . v 2 i 1.167$

\section{RESUMO}

O presente artigo tem por objetivo a discussão acerca da relação entre o processo de formação e atuação médica e o desenvolvimento da ideação suicida. Para tanto, fez-se uma pesquisa bibliográfica com diversos autores como, Alberto Manuel Quintana, Sérgio Baldassin, Nathália Della Santa e Antonio Ivo de Carvalho contribuíram para a construção dessa reflexão, por meio de dados, revisões literárias e análise de documentações. Dessa forma, espera-se que o público compreenda a gravidade e amplitude do problema em questão.

Palavras chave: Depressão. Medicina. Suicídio.

\section{ABSTRACT}

This article aims to discuss the relationship between the process of training and medical practice and the development of suicidal ideation. For this, a bibliographic research was made with several authors such as Alberto Manuel Quintana, Sérgio Baldassin, Nathália Della Santa and Antonio Ivo de Carvalho contributed to the construction of this reflection, through data, literary reviews and documentation analysis. Thus, the public is expected to understand the seriousness and breadth of the problem at hand.

Keywords: Depression. Medicine. Suicide.

\section{INTRODUÇÃO}

Este artigo apresenta os resultados sobre a questão do suicídio envolvendo estudantes de Medicina e médicos. Esse assunto é frequentemente mascarado e tratado como um tabu, o que impede a sua discussão na sociedade em geral. Ansiedade e depressão são duas patologias recorrentes em indivíduos com ideação suicida, e o fato de serem negligenciadas pela população corrobora com o aumento dos índices de episódios suicidas (SANTA e CANTILINO, 2016).

A oferta de programas criados para abordar atitudes em relação à saúde mental, também é importante para conscientizar o estudante de que o próprio curso pode contribuir para gerar, manter ou desencadear transtornos mentais ou de comportamento, como o uso problemático de álcool. Dado que a depressão e suas consequências, como o suicídio, são transtornos comuns geralmente associados a quadros ansiosos, e mais frequentes nos estudantes de medicina que na população 
em geral, faz-se necessária a oferta precoce de atendimento e apoio (BALDASSIN, 2006).

\section{METODOLOGIA}

A metodologia utilizada baseou-se em documentos, artigos, sites, dados e nas contribuições teóricas de renomados autores, como Antônio Ivo de Carvalho e Sergio Baldassin, auxiliaram na construção desse projeto, caracterizando-se uma revisão bibliográfica. Os autores abordam pontos importantes sobre fatores que conduzem os estudantes e médicos ao desenvolvimento de um pensamento suicida. Para tanto, em suas obras, utilizam como base outros importantes teóricos, também citados ao longo do texto para contribuir em sua construção. Como base de pesquisa foi utilizado as plataformas "PubMed" e "Google Acadêmico".

\section{RESULTADOS E DISCUSSÃO}

O processo saúde-doença é conhecido há anos, e reproduzido na sociedade por fortes processos de determinação social, econômica, ambiental e cultural. A criação da comissão global sobre determinantes sociais da saúde - 2003 - iniciou um processo de sistematização do conhecimento disponível e de articulação de iniciativas e do estímulo de políticas inspiradas nesse modelo (DE CARVALHO, 2013).

Ainda segundo o mesmo autor, a maioria das doenças que se tem conhecimento está intimamente ligada às condições em que as pessoas nascem, vivem, trabalham e envelhecem. Esses fatores são influenciados pelo posicionamento social dos indivíduos que, em muitos casos, levam às injustiças na área da saúde. "A saúde não é mercadoria transacionável no mercado; é uma questão de direitos e um dever do setor público. Como tal, os recursos para o setor devem ser equitativos e universais (DE CARVALHO, 2013, pág. 27)". Considerar que as condições ambientais interferem nesse processo é um ponto importante, para a interpretação e intervenção no processo saúde e doença.

Compreender essa abordagem na sociedade de modo geral, já é um obstáculo, quando se menciona tal assunto envolvendo a área da saúde, mais especificamente a formação e atuação médica, a situação se torna mais agravante. $O$ fato de, historicamente ser construída uma imagem de onipotência sobre a figura do médico, este acaba sendo negligenciado como um ser psicossocial, que também necessita de cuidados (BALDASSIN, 2006).

Essa doutrina já começa a ser construída, antes mesmo do ingresso no ensino superior, no processo de seleção para o curso. Essa leva em consideração apenas a capacidade intelectual e a bagagem teórica, desconsiderando outros fatores cruciais que refletem no sucesso acadêmico e profissional do médico como a sociabilidade, empatia e tolerância à desapontamentos. Além disso, passam por um dos vestibulares mais concorridos do país - um dos reflexos da superestimação da profissão - devido à idealização da população, em virtude da crença no retorno econômico e status social que a profissão supostamente proporcionará (SANTA e CANTILINO, 2016). Conforme Quintana (2008), a imagem mais associada ao médico, quase que um estereótipo, é a do herói conquistando a morte, sendo visto pela população em geral como uma figura que serve como sinônimo de sucesso e autossuficiência.

No início do curso, o primeiro obstáculo apresenta-se como a mudança súbita de hábitos, principalmente durante o primeiro semestre. A expectativa construída 
pelos estudantes em virtude do estereótipo envolvendo o curso no qual estão inseridos é quebrada quando, ao ter os primeiros contatos com a grade curricular se deparam com matérias extremamente teóricas que pouco se relacionam a medicina propriamente dita - contato com pacientes e patologias - essas frustações desencadeiam os primeiros traços de ansiedade. Em contrapartida, quando começam a vivenciar a rotina hospitalar, são inseridos em uma realidade que desfaz a ideia de onipotência, pois se veem impotentes frente a casos clínicos que extrapolam a medicina, em situações onde a patologia é irreversível, ou quando o tratamento não depende somente da equipe médica como também dos sistemas de saúde, a exemplo do SUS (Sistema Único de Saúde) (QUINTANA, 2008).

Ao longo desse processo, a instituição centraliza sua atenção na formação técnica do acadêmico, menosprezando sua saúde mental, por se acreditar que a manifestação emocional prejudicaria o desempenho profissional. O método de ensino ativo PBL (Aprendizado Baseado em Problemas) que vem sendo implementado nas instituições, preocupa-se com a despersonalização do paciente pelo profissional, mas essa atenção não é a mesma quando esse fato ocorre com o estudante de medicina. O resultado é a formação de um profissional que domina todos os conhecimentos das áreas biomédicas, mas não é capaz de reagir as suas próprias angústias, afetando a sua eficácia na relação médico-paciente (SANTA e CANTILINO, 2016).

A OMS (Organização Mundial da Saúde) considera o suicídio como um problema de saúde pública, visto que é uma das principais causas de morte na maioria dos países. Ainda segundo o órgão, nos 45 anos o perfil suicida migrou da faixa mais idosa para faixas mais jovens (SANTA e CANTILINO, 2016).

Segundo Santa (2016), o comportamento suicida e dividido em: ideação suicida, tentativa de suicida, e suicídio consumado. Ideias, desejos, ilusões, fantasias envolvendo a morte como solução para um problema que aparenta ser irreversível. As intensidades desses comportamentos têm correlação em muitos casos com práticas masoquistas. A autoflagelação se apresenta como uma válvula de escape, uma vez que dores psicológicas são substituídas por dores físicas. A gravidade dessas ações varia com as sucessivas tentativas de suicídio, que aumentam o risco de 0 ato ser concluído. Frente a esse contexto, quando direcionamos esse assunto para área médica, as taxas de suicídio são mais elevadas que na população em geral.

\begin{abstract}
Uma pesquisa da Universidade de Oxford publicada em 2004 investigou fatores relacionados ao suicídio em médicos. A pesquisa foi realizada por meio de autópsia psicológica de 38 médicos que cometeram suicídio. Dentre esses, 25 apresentavam algum tipo de transtorno mental, principalmente depressão e abuso de álcool. Os problemas enfrentados por esses médicos foram identificados como relacionados ao trabalho (25), aos relacionamentos interpessoais (14), financeiros (10) e a um conjunto desses fatores (12) (SANTA e CANTILINO, 2016, pág. 775).
\end{abstract}

O curso de medicina é reconhecido por desencadear estresse constante e sobrecarga intelectual em seus graduandos, o que se torna pré-requisito para uso de substâncias estimuladoras e transtornos depressivos, os quais são fundamentados na dificuldade de corresponder à representação social do médico. Esses problemas refletem no bem-estar físico e no desempenho dos acadêmicos (QUINTANA, 2008).

A depressão, doença muito estudada no meio médico, é, por sua vez, a principal patologia psiquiátrica que acomete os próprios alunos e profissionais da área (ALVES, 2010). 
Além da vulnerabilidade psicológica que cerca essa classe de profissionais, fatores externos como a dependência financeira, pressão por alto rendimento, intensa atividade intelectual, sobrecarga horária e distúrbios de identidade narcisista são, de fato, contribuintes, quando aliados à sensibilidade psicológica, na decisão de cometer suicídio. Pontuam-se também como facilitadores do ato a disponibilidade de meios (na maioria dos casos o uso de fármacos) e conhecimento científico do funcionamento fisiológico do corpo humano, que são adquiridos ao longo da graduação (DE CARVALHO, 2013).

Um estudo realizado com estudantes de Berlim em 2010 demonstrou que cerca de um terço dos 850 alunos participantes da pesquisa usa algum tipo medicação de forma regular como uma fuga dos problemas através de fármacos (SANTA e CANTILINO, 2016, pág. 776).

A morte é tratada como uma das principais temáticas dentro do curso de Medicina e ao longo da carreira profissional do médico. É dever deste profissional prevenir e tratar seus pacientes visando deixá-los afastados de situações que os levem ao fim de suas vidas. Dessa forma, a construção de um pensamento suicida na figura de estudantes ou dos profissionais vai contra os princípios que norteiam suas ações (SANTA e CANTILINO, 2016).

Nota-se que, dados alarmantes, que superam os da população em geral, são insuficientes para discussão acerca do suicídio, em razão de esse ser tratado como tabu pela sociedade em geral. Devido a esse fator torna-se um desafio aos profissionais médicos lidar com os seus próprios problemas psicológicos (ideações suicidas), mesmo sendo um assunto comumente vivenciado e tratado na área da saúde. A imagem de equilíbrio e disponibilidade assumida pelo médico dificulta a aceitação e consequente solicitação de ajuda (SANTA e CANTILINO, 2016).

Recomenda-se o envolvimento dos estudantes em pesquisas sobre transtornos depressivos e ansiosos, como forma de iniciá-los no reconhecimento e manuseio desses distúrbios, desprezados com frequência no meio acadêmico e profissional (BALDASSIN et al., 2006, pág. 30 ).

Desde o início do curso, os acadêmicos devem se envolver em atividades acerca das dificuldades impostas ao longo de sua trajetória dentro da instituição, a fim de diminuir as chances de desenvolver algum distúrbio psíquico (BALDASSIN, 2006).

Como na população geral, encontram-se fatores importantes com relação a idade, sexo, profissão, estado físico e fatores psicossociais. Entre os médicos que cometeram suicídio, colegas próximos têm referido mudança no comportamento e aumento de indecisão, desorganização e depressão por dois ou quatro meses precedendo o suicídio, como na população geral (SAKINIFSKY, 1980).

Apresenta dados importantes: suicídio entre médicas é mais frequente que entre médicos; a taxa de suicídio entre as médicas é quatro vezes maior que nas mulheres da população geral com mais de 25 anos, nos Estados Unidos; a incidência de morbidade psiquiátrica, alcoolismo e uso de drogas é alta nos médicos suicidas; a disponibilidade e a oportunidade de acessos a meios de suicídio (drogas, venenos, etc.) são maiores na população médica.

Ainda segundo o mesmo autor, atribui-se a alta incidência de suicídio nas mulheres pela personalidade que as levam a escolher a carreira médica por serem competitivas, ambiciosas, compulsivas, individualistas e inteligentes. Elas podem escolher a carreira médica para competir com homens ou para não competir com as 
mulheres. Outro indicativo de suicídio entre médicas e químicas está no fato de que elas têm, sempre, não só a oportunidade de acesso a drogas e venenos letais, mas, também, o conhecimento científico, se comparadas às enfermeiras e professoras. $O$ mesmo se verifica com o grupo masculino, neste último aspecto, isto é, o acesso fácil e o conhecimento dos meios letais (ROSS, 1973).

Esse aumento do nível de estresse, levando em conta os conhecimentos atuais de mecanismos de adaptação, podem levar estudantes de medicina a agirem com estratégias, muitas vezes inadequadas, para compensação (mecanismos de coping stress) através da fantasia (com queda do rendimento), busca de alivio (uso de substância) ou fuga. A escola médica deve estar preparada no auxílio do estudante no gerenciamento do estresse. Abordagens cognitivocomportamentais e grupos terapêuticos podem ser úteis na redução do impacto do estresse do curso médico. Os benefícios percebidos pelos estudantes submetidos a programas de gerenciamento de estresse são vários, entre eles: aumento da produtividade e do foco, melhora da capacidade de empatia, redução dos sintomas de ansiedade e depressivos, e maior leque de habilidades e competências para lidar com eventos estressantes no futuro (VAN NGUYEN, 2014).

Diversos estudos têm sugerido que determinadas especialidades médicas podem aumentar a taxa de suicídio: anestesistas, psiquiatras, oftalmologistas e patologistas são referidos como os mais vulneráveis (MELEIRO, 1998).

$O$ fato de o psiquiatra não dispor de recursos facilitadores do diagnóstico, tais como o raio- $\mathrm{X}$, eletrocardiograma, exames laboratoriais e outros - além dos aspectos sociais dos pacientes com os quais tem que lidar, da transferência no ato psicoterapêutico e, algumas vezes, da oposição da família e do próprio paciente, sem crítica do seu estado mórbido - proporcionaria uma "existência corrosiva" que acabaria vendo o suicídio como uma saída dessa corrosão (WEKSTEIN, 1979).

Aponta-se como fatores relevantes o conhecimento farmacológico que o médico possui, tornando qualquer tentativa de suicídio altamente letal, e o uso abusivo de drogas e álcool nessa população, confirmado pelo elevado índice de cirrose entre médicos ingleses e do País de Gales (SAKINIFSKY, 1980).

Segundo o mesmo autor, a ingestão excessiva de drogas é uma forma de suicídio muito aceita culturalmente, sendo os medicamentos, principalmente os psicofármacos, utilizados na maioria dos suicídios de médicos. Segundo Simon, em 1968, realizando um estudo retrospectivo em 62 escolas médicas norte-americanas e três canadenses, concluiu que o suicídio é a segunda causa de morte entre os estudantes de medicina, perdendo apenas para os acidentes. Os dados encontrados com os alunos da Faculdade de Medicina da Universidade de São Paulo aproximaram-se aos dos obtidos por Simon. Não há estudos em outras faculdades de medicina do Brasil sobre esse assunto.

No Brasil, Martins destaca alguns fatores estressantes associados ao exercício profissional: sobrecarga horária, privação de sono, comportamento idealizado contato intenso e freqüente com a dor e o sofrimento; lidar com a intimidade corporal e emocional - contato com a morte e com o morrer; lidar com pacientes difíceis incertezas e limitações do conhecimento médico, isto é, o medo do erro médico (MARTINS, 1991).

Pouco tem sido publicado sobre intervenção de assistência a esses grupos de pessoas com trauma de membros que se suicidaram. Suicídio pode provocar reações de choque e sentimentos contraditórios: raiva, culpa, medo e tristeza, e pode resultar 
em uma disfunção organizacional. A natureza e a intensidade das emoções podem provocar e limitar as suas expressões conscientes (MELEIRO, 1998)

Ainda segundo o mesmo autor, vários estudos mostram que cerca de dois terços dos indivíduos que se suicidam comunicaram suas intenções previamente para a família, amigos ou médicos, o que demonstra a importância de se ter uma comunidade no meio acadêmico — bem como no meio médico — receptiva e com um papel facilitador da busca de ajuda preventiva.

Quando divididos por ciclos, os estudos apontam que o ciclo pré-clínico e o internato como momentos de maior risco. Mas a questão do porquê os estudantes de medicina têm maior risco ainda não foi completamente esclarecida. Devemos levar em conta que a Depressão é um quadro multifatorial, que inclui desde fatores genéticos como ambientais em sua fisiopatologia. Em alguns casos, os aspectos genéticos parecem ser predominantes (presença de diversos membros afetados na família), em outros aspectos ambientais parecem ter um papel relevante, bem como capacidade de gerenciamento destes fatores (SILVA, 2009).

\section{CONSIDERAÇÕES FINAIS}

Em razão de estarem psicologicamente abalados, em virtude do contato diário com a morte, com patologias irreversíveis ou casos clínicos de difícil compreensão, tanto estudantes como médicos encontram-se mais sensibilizados ao desenvolvimento de doenças, principalmente a depressão. Essa, por sua vez, influencia diretamente na ideação suicida e, se tratada como um tabu, torna-se de difícil diagnóstico e tratamento. Portanto, é nítida a necessidade de tratar do assunto durante a graduação e estar em constante acompanhamento dos profissionais e da instituição, pois o tratamento precoce dos comportamentos suicidas evita a finalização do ato em si. É importante que, além de um profissional que domine os conhecimentos técnicos tenhamos um profissional saudável, apto a lidar com as situações-problema a que seja apresentado.

Avaliar um paciente suicida é complicado pelo fato de despertar, com freqüência, fortes sentimentos no médico examinador, principalmente ansiedade por um erro de conduta e uma conseqüência catastrófica. O médico deve estar calmo, pois os suicidas aumentam na mesma proporção das reações negativas do entrevistador em relação ao paciente. Deve evitar reações moralistas e críticas. 0 suicídio é um assunto extremamente pessoal, e o paciente deve ter oportunidade de conversar a sós com o médico, pois, geralmente, sente-se aliviado ao ser questionado sobre o assunto. Todas as ameaças de suicídio devem ser encaradas com seriedade, mesmo que possam parecer falsas (manipulação). A escolha da conduta do tratamento dependerá das impressões gerais do médico quanto ao risco de suicídio num futuro próximo e à necessidade de tratamento dos problemas psiquiátricos.

Para o médico já em exercício da profissão, um programa de conscientização e orientação de que a informação técnica anteriormente adquirida não lhe dá imunidade aos conflitos emocionais. Publicações constantes para familiarização por parte dos colegas médicos com a profilaxia e reconhecimento dos sinais preditivos de suicídio. Desenvolvimento de uma assistência psiquiátrica e psicoterápica para médicos em risco de suicídio. Preparo de profissionais para lidar com esse grupo de pacientes, pois os sentimentos positivos e negativos da contratransferência se misturam, principalmente os de identificação. É imperativo que a equipe defina sua 
relação com o paciente-médico e enfatize a sua responsabilidade de cooperação, evitando privilégios especiais. Pensamos ser importante que a equipe não seja familiar (conhecida) do paciente médico.

\section{REFERÊNCIAS}

ALVES, João Guilherme Bezerra. Qualidade de vida em estudantes de Medicina no início e final do curso: avaliação pelo Whoqol-bref. Revista brasileira de educação médica. Instituto de Medicina Integral Prof. Fernando Figueira. Recife. 2010.

BALDASSIN, Sergio, et al. Traços de ansiedade entre estudantes de medicina. Arquivos médicos do ABC. Vol. 31. São Paulo. 2006.

DE CARVALHO, Antônio Ivo. Determinantes sociais, econômicos e ambientais da saúde. In: FUNDAÇÃO OSWALDO CRUZ. A saúde no Brasil em 2030 - prospecção estratégica do sistema de saúde brasileiro: população e perfil sanitário. Vol. 2. Rio de Janeiro. 2013.

QUINTANA, Alberto Manuel. A angústia na formação do estudante de medicina. Revista brasileira de educação médica. Vol. 32. Rio de Janeiro. 2008.

SANTA, Nathalia Della. Suicídio entre Médicos e Estudantes de Medicina: Revisão de Literatura. Revista brasileira de educação médica. Universidade Federal de Pernambuco, Recife. 2016.

ROSS, Mathew. Suicide among physicians: a psychological study. Dis Nerv System, Chestnut Hill, Massachusetts, Estados Unidos. 1973.

MELEIRO, Alexandrina Maria Augusto da Silva. Suicídio entre médicos e estudantes de medicina. Revista Associação Médica Brasileira. Vol. 44, n. 2, p. 135-140. São Paulo. 1998.

MELEIRO, Alexandrina Maria Augusto da Silva, WANG, Yuan Pang. Suicídio e tentativa de suicídio. Psiquiatria básica. Porto Alegre.1995.

WEKSTEIN, Louis. Handbook of suicidology. Nova lorque. 1979.

SIMON, Harold. Mortality among medical students. J Med Educ. Nova lorque. 1968.

MARTINS, Lan. Atividade médica: fatores de risco para a saúde mental do médico. Rev Bras Clin Terap. São Paulo. 1991.

VAN NGUYEN, Hien, et al. The relationships between the use of self-regulated learning strategies and depression among medical students: an accelerated prospective cohort study. Psychol Health Med. Nova lorque. 2014.

SILVA, Clóvis Alexandrino, et al. Suicidal ideation among students enrolled in healthcare training programs: a cross-sectional study. Revista Brasileira de Psiquiatria. São Paulo. 2009. 